\title{
LE DROIT FRANÇAIS ET LE COUPLE
}

\author{
Anne Cathelineau-Roulaud ${ }^{1}$ \\ Habilitée à diriger des recherches, Université de la Rochelle, France \\ email: anne.cathelineau@univ-lr.fr
}

CATHELINEAU-ROULAUD, Anne. Le droit français et le couple. International and Comparative Law Review, 2013, Vol. 13., No. 1, pp. 171-183.

DOI: 10.1515/iclr-2016-0067.

\begin{abstract}
French family law has just undergone tremendous changes. The may 17 Act which allows same sex marriage means the acknowledgement of a right for couples either homosexual or heterosexual. These couples can be married or not. Beyond the diversity, we can however feel the emergence of a right for the couple, that is to say common rules for all couples. The couple, from every angle, became an essential and inescapable element of family law which has its proper rules.
\end{abstract}

Keywords: Couple, Homosexual, Heterosexual, Marriage, PACS, Cohabitation, Filiation

Le couple est aujourd'hui sous les feux de l'actualité en droit français ! $\mathrm{Au}$ terme de débats houleux, la loi du 17 mai 2013 ouvrant le mariage aux couples de même sexe $e^{2}$, validée par le Conseil constitutionnel ${ }^{3}$ invite en effet à s'interroger sur la notion même de couple et sur la façon dont le droit français appréhende cette notion.

S'il est acquis que pour former un couple il faut être deux, le mot couple étant issu du latin «copula » qui signifie « tout ce qui sert à attacher, lien, chaîne ${ }^{4}$, le couple n'est pourtant pas le mode exclusif de la vie à deux : la vie à deux peut être conçue déchargée de son aspect charnel. La jurisprudence récente de la Cour européenne des droits de l'homme s'est d'ailleurs clairement engagée dans cette voie ${ }^{5}$.

1 Anne Cathelineau-Roulaud est une Maître de conférences à l'Université de la Rochelle; Habilitée à diriger des recherches (HDR); Membre du Centre d'Etudes Juridiques et Politiques de la Rochelle (CEJEP). Email: anne.cathelineau@univ-lr.fr

2 L. $n^{\circ}$ 2013-404, 17 mai 2013 : JO 18 mai 2013, p. 8253. Loi ouvrant le mariage aux personnes de même sexe : JCP G 2013, 590; V. AVENA-ROBARDET, La réforme du mariage : un dossier complet : $\mathrm{n}^{\circ}$ spécial AJ famille juin 2013.

3 Cons. Const., déc, n² 2013-669 17 mai 2013 DC.

4 Dictionnaire latin-français Félix Gaffiot: Hachette

5 Arrêt Burden/ Royaume Uni, CEDH, 29 avril 2008 : www.rtdh.eu/pdf/burden-c-royaumeuni-20080429.pdf : "Le lien unissant deux sœurs en dépit de leur vie commune ne peut être 
Il apparait en tous cas que ce sont les sociologues plus que les juristes qui ont souligné d'abord l'importance du concept. Ce dernier ne fait en effet l'objet d'aucune définition légale ${ }^{6}$. En droit, il semble d'ailleurs que le terme soit d’abord entré dans le langage juridique par la porte du droit fiscal afin de faire supporter une imposition globale par les couples mariés puis par la porte du droit social, celui-ci ne distinguant pas selon la nature du couple, marié ou vivant en concubinage. En droit civil, la notion de couple est apparue plus tardivement avec les lois dites de bioéthique du 29 juillet 1994 qui y font référence en particulier pour préciser les conditions de l'assistance médicale à la procréation et envisagent à ce titre le couple comme formé d'un homme et d'une femme. La Loi du 15 novembre 1999 relative au PACS a par la suite utilisé le terme pour définir le concubinage, et faire de la « vie en couple» une condition dapplication de cette notion.

A l'heure actuelle, en sociologie comme en droit, un couple semble devoir être compris comme un ensemble de deux personnes, liées par une volonté de former une communauté matérielle et affective et se traduisant par des relations charnelles. «Vivre en couple, c'est, pour reprendre une expression de Victor Hugo, être deux et nềtre qu'un"!

Lappréhension du couple par le droit français concerne essentiellement le droit civil ${ }^{7}$ et plus particulièrement le droit de la famille. Le couple apparaît en effet comme une composante majeure du droit de la famille, le concept de famille étant toutefois une notion qui ne fait elle-même l'objet d’aucune définition légale. Conçue comme « lélément naturel et fondamental de la société » par la Déclaration universelle des droits de l'homme de 1948, elle recouvre à l'heure actuelle des réalités très variées. Si la famille contemporaine est formée du couple de parents et des enfants se démarquant de la famille primitive plus large fondée sur le lignage ${ }^{8}$, elle est susceptible d’englober aussi les familles monoparentales qui se développent d'ailleurs avec l'accroissement à la fois des divorces sans remariage et des maternités sans mariage. Elle est alors réduite à l'extrême : un seul parent, et souvent un seul enfant. Les conceptions de la famille ont ainsi évolué selon les temps, les époques et les sociétés et il apparaît que notre société occidentale contemporaine se caractérise par le pluralisme.

En dépit de cette hétérogénéité des modèles familiaux, la famille souche fondée sur le couple semble être prévalente. Le lien familial créé par le couple

assimilé au lien unissant les membres d'un couple, qu'ils soient conjoints ou partenaires civils".

6 La plupart des dictionnaires juridiques ignorent d'ailleurs cette notion. V. cependant G. CORNU, Vocabulaire juridique : Ass. Henri Capitant, PUF 2011, qui donne la définition suivante « Union que forme un homme et une femme entre lesquels existent des relations charnelles et en général une communauté de vie, soit en mariage, soit hors mariage; se dit parfois de deux individus de même sexe qui vivent ensemble».

7 V. J.- J LEMOULAND, Le couple en droit civil : Dr. famille 2003, chron. 22.

8 L'histoire de la famille en Occident illustre une évolution que DURKHEIM appelait la «loi $d u$ rétrécissement continu de la famille». 
est certainement un facteur d'équilibre au sein de notre société qu'il s'agisse du couple conjugal ou du couple parental.

Etudiée ainsi dans le cadre du droit français de la famille, force est de constater que le couple est, paradoxalement, sans faire l'objet d'une définition légale précise, utilisé par la plupart des ouvrages contemporains de droit de la famille comme critère de subdivision. On peut dès lors s'interroger sur les raisons de cet engouement. Une première explication paraît être d'ordre historique. En droit canonique en effet, la corpula carnalis est un élément indispensable à la perfection du mariage ${ }^{9}$. Le terme est ainsi resté familier et passé dans le langage courant. Une autre justification peut être trouvée dans la volonté d'une bonne partie de la doctrine juridique contemporaine de tenter de reconstruire le droit de la famille sérieusement mis à mal par la multitude des réformes récentes autour de cette notion de couple, une reconstruction ne pouvant se faire sans fondation et le couple étant alors considéré comme un élément fondateur de la famille.

Le sujet présente de nombreux intérêts qui méritent d’être évoqués.

Il revêt d'abord un intérêt indéniable d'actualité lié à la loi nouvellement votée dans un climat agité et aux répercussions de cette loi largement discutées. Mettant en œuvre l’engagement de campagne 31 de l'actuel Président de la République française François Hollande, ce texte a été annoncé par son premier ministre Jean-Marc Ayraut comme " une évolution majeure de notre Code civil, une décision de justice et d'égalité qui prend acte de l'évolution de notre société ». A vrai dire, ce texte nouveau constitue comme ont pu le constater certains auteurs une véritable révolution et non une simple évolution, car il concerne non seulement le mariage mais également la filiation, ouvrant la possibilité d'adoption d'un enfant par des couples mariés de même sexe, ce qui marque une véritable rupture sociale. En consacrant une nouvelle conception du mariage et donc du couple marié, le texte bouleverse inévitablement l'ensemble du droit de la famille français ${ }^{10}$.

Le sujet présente en outre un intérêt de droit comparé, bon nombre de législations étrangères ayant autorisé le mariage homosexuel ces dernières années : les Pays- Bas en 2000, la Belgique en 2003, l'Espagne et le Canada en 2005, la Norvège et la Suède en 2008 et en 2009, le Portugal, le Luxembourg, l'Islande, l'Argentine et le Mexique en 2010, le Danemark et l'Uruguay en 2012. Par ailleurs, plusieurs Etats des Etats Unis l'ont également adopté. Ce constat ainsi que celui de l'ouverture en général à ces couples du droit d'adopter traduit en effet une convergence intéressante du droit français avec les droits en cause invitant à s'interroger sur le sens d'une telle évolution ${ }^{11}$.

9 V. nouveau C. dr. canonique, canon 1061 : c'est le rapport charnel qui consomme le mariage et le rend indissoluble.

10 H. FULCHIRON, La reconnaissance de la famille homosexuelle : étude d'impact : D. 2013, p. 100.

11 S. PARICARD, Mariage homosexuel et filiation. Quelques éléments de droit comparé : Dr. 
On soulignera en dernier lieu l'intérêt pratique du sujet au-delà de son aspect théorique. La façon dont le droit français appréhende aujourd'hui le couple n'est pas en effet sans provoquer un certain nombre de conséquences pratiques qui ne manquent pas d'intéresser les praticiens tels que les notaires par exemple, conseillers des familles par excellence ${ }^{12}$. Louverture du mariage et de l'adoption aux couples de même sexe suppose en effet une adaptation du droit des régimes matrimoniaux, du droit des successions ${ }^{13}$ et du droit du nom notamment ${ }^{14}$.

Létude du droit français et du couple a vocation à analyser l'évolution de la notion de couple et des règles qui le concerne, l'objectif étant de mesurer l'impact de ce concept dans le droit de la famille français actuel. Il ne sagit pas de procéder à une analyse descriptive qui serait purement technique mais à une étude démonstrative amenant à dégager un certain nombre de fils conducteurs.

Si l'évolution du droit français traduit ainsi la reconnaissance d'un droit des couples (I), la notion de couple se déclinant en plusieurs variantes, on peut y percevoir aussi l'émergence d'un droit du couple (II), c'est-à-dire d'un socle commun protégeant l'ensemble des couples dans leur diversité, dans l'intérêt des familles.

\section{LA RECONNAISSANCE D'UN DROIT DES COUPLES}

Lévolution contemporaine du droit de la famille amène à distinguer deux façons d'appréhender le couple, chacune de ces façons revêtant elle-même plusieurs formes. Le terme de couple sert en effet à désigner l'union de deux personnes, mais il est parfois employé aussi pour qualifier la relation qui existe entre les parents vis-à-vis de leurs enfants.

Dans le premier cas, c'est le couple conjugal (A) qui est en cause, tandis que dans le second il s'agit du couple parental (B).

\section{A. La conjugalité}

Le couple conjugal est aujourd'hui pluriel dans son mode d'expression. Le mariage n'est plus qu'une forme de couple parmi d'autres et la conjugalité déborde le cadre du mariage.

famille, janvier 2013, dossier 8; C. BEAUDOUIN, La loi française peut-elle contribuer au renversement du consensus européen ?: AJ Famille 2013, p. 366.

12 J. HAUSER, Le mariage des couples de même sexe et le notaire : une réforme par prétérition et procrastination : Dr. famille 2013, dossier 7.

13 C. PERES, Le droit des successions dans le projet de loi ouvrant le mariage aux couples de personne de même sexe : Dr. famille 2013, dossier 6.

14 C. DOUBLEIN, Le nom de famille : les modifications apportées par la loi du 17 mai 2013 : AJ Famille 2013, p. 349; F. GRANET-LAMBRECHTS et P. HILT, Les incidences sur létat civil des époux de la loi du 17 mai 2013 ouvrant le mariage aux personnes de même sexe : AJ Famille 2013, p 336; Mariage pour tous : publication des dispositions réglementaires : Dalloz actualité 29 mai 2013. 
A cet égard, lévolution a été remarquable car pendant fort longtemps, le seul couple envisagé par la loi était le couple marié. Le mariage était la seule forme d'union reconnue et organisée non seulement par le droit canon qui régissait seul les relations familiales sous l'Ancien Régime, mais aussi par le Code civil qui laissait délibérément hors de son champ le concubinage. La formule restée célèbre de Bonaparte à cet égard était "Les concubins se passent de la loi, la loi se désintéresse d'eux ". Le couple marié constituait le socle de l'édifice familial : pas de famille sans couple et pas de couple sans mariage. Cette famille issue du mariage était dotée d'un chef, le mari, la femme initialement incapable étant soumise à son autorité.

C'est en fait dans la seconde partie du XXe siècle qu'un mouvement de libéralisation s'est amorcé et a fait céder cette exclusivité. Il s'agit des années 60 à 90 que l'on peut appeler les années Carbonnier du nom de l'initiateur de la plupart des réformes de leepoque. Un vent de liberté et d'individualisme souffle alors sur le droit de la famille!

Le couple n'a plus de chef : les époux sont égaux et le mariage voit son caractère institutionnel décroître, la liberté du divorce devenant le corollaire de la liberté du mariage. Le mariage n'est plus le seul moyen de fonder une famille.

Les couples hétérosexuels non mariés ont d'abord été pris en compte par le droit de manière parcellaire. L'ignorance juridique du concubinage a fait place peu à peu à des textes particuliers attachant certains droits à des situations de concubinage (en matière de bail, de responsabilité, de sécurité sociale...). Et le législateur a fini par donner lui-même une définition légale du concubinage faisant sortir de la sorte les concubins du non-droit (article 515-8 du Code civil). Puis, la loi du 15 novembre 1999 a offert un cadre juridique à ceux qui souhaitent organiser leur union sans vouloir ou pouvoir s'inscrire dans le moule strict du mariage. C'est l'introduction du PACS en droit français (pacte civil de solidarité), l'article 515-1 du Code civil définissant le PACS comme " un contrat conclu par deux personnes physiques majeures, de sexe différent ou de même sexe, pour organiser leur vie commune ».

Les couples homosexuels par la suite se sont vus avec le PACS faire lobjet d'une reconnaissance légale. Même s'il ne leur est pas finalement dédié, le PACS est né à la suite des revendications matrimoniales des couples homosexuels. Le PACS connaît un succès grandissant car c'est un contrat dont la formation et la résiliation paraissent fort souples (on compte aujourd'hui 2 PACS pour 3 mariages). Les couples de partenaires ne forment plus une communauté marginale et sont pour la plupart hétérosexuels. Et, la conclusion d'un PACS correspond assez souvent aujourd'hui à un préliminaire du mariage (ce que l'on appelait autrefois les fiançailles).

La loi du 17 mai 2013 ouvrant le mariage aux couples de même sexe amène en dernier lieu à offrir le cadre légal bien connu du mariage aux couples d'homo- 
sexuels Le mariage homosexuel est célébré dans les mairies de France depuis le 29 mai. L'exposé des motifs présenté par Madame Christine Taubira, Garde des sceaux, Ministre de la Justice révèle la volonté de franchir une nouvelle étape après l'adoption de la loi relative au pacte civil de solidarité, afin de répondre à la demande des couples de même sexe qui souhaitent pouvoir se marier et à leur demande d'accès à l'adoption.

Ouvrir le mariage aux couples homosexuels conduit inévitablement à repenser aujourd'hui la conjugalité. En ouvrant le mariage aux personnes de même sexe, on modifie en effet la définition du mariage. Le mariage homosexuel fait voler en éclats le mythe du mariage - berceau de la famille fondée sur les liens du sang. Le mariage ne constitue plus une union sexuée creuset de la procréation ; il apparait être un statut du couple parmi d'autres. Larticle 143 nouveau du Code civil est ainsi rédigé de la façon suivante «Le mariage est contracté par deux personnes de sexe différent ou de même sexe ». Larticle 144 quant à lui ne contient plus de référence sexuée à l'homme et la femme mais se contente de déterminer lâge nuptial «Le mariage ne peut être contracté avant dix huit ans révolus ». Le texte ne modifie pas le régime juridique du mariage. Certaines dispositions traditionnelles sont apparues toutefois inadaptées, comme celles relatives au nom de famille, le nom du père ne pouvant plus être imposé. Par ailleurs, de nombreuses dispositions de coordination ont à cet égard été prévues, les mots de " père » et « mère » étant remplacés par ceux de parents, et les mots de " mari » et «femme " par ceux dépoux.

\section{On aboutit ainsi aujourd'hui à une trilogie des couples.}

Il y a d'abord le mariage, contrat et de moins en moins institution dont la nature originale est la marque de la durée. Union durable, elle est organisée par la loi à travers un statut complet, fait de droits mais aussi dobligations. Le mariage est affaire de personnes et de biens : il fait lobjet d'un statut patrimonial contenu dans le droit des régimes matrimoniaux. On peut simplement se demander comment le droit du mariage construit à la base pour des couples composés s'un homme et d'une femme pourra s'adapter à des couples composés de deux hommes ou de deux femmes. Le refus de la discrimination impose de traiter à égalité de droits des situations identiques. Or, comment considérer comme identiques des situations qui à la base ne le sont pas ? Certains critiquent cette évolution estimant que le terme de mariage est par là même dénaturé15. Il est vrai que le mariage perd sa dimension institutionnelle destinée à offrir à l’enfant sa double ascendance maternelle et paternelle pour se réduire à un contrat d'union civile régissant la vie à deux, dont les règles impératives relèvent d'un ordre public social et non plus familial, simplement déclenché par la cohabitation. Le nouveau texte devra en tous cas amener à informer particulièrement

15 Un auteur s'interroge sur l'avenir de l'institution, se demandant si elle reste une institution ou si elle n'est pas devenue une auberge espagnole où chacun entre et sort au nom de ses droits subjectifs.; V. J. CASEY, Quel avenir pour le mariage : Gaz. Pal 2012, p. 9, nº 259. 
les couples de même sexe des effets qui s'appliqueront au choix de la structure mariage, cest-à-dire le régime matrimonial qui en découle, le droit du divorce qui pourrait s'appliquer avec notamment le risque d'une prestation compensatoire et les conséquences particulièrement importantes des réformes récentes sur la condition du conjoint survivant.

Outre le mariage, il y a aussi le PACS qui correspond également à un couple contractuel mais selon une organisation légale plus libertaire. Il n’a pas la même solennité que l'acte de mariage passé devant un officier d'état civil. Différemment, il fait en effet l'objet d'un acte sous seing privé ou d'un acte notarié ${ }^{16}$ et d'une déclaration puis d'un enregistrement soit par le greffier du tribunal d'instance si lacte est sous seing privé soit par devant notaire si l'acte est notarié, ceux-ci faisant alors procéder aux formalités de publicité ${ }^{17}$. Aucun passage n’est prévu par devant l'officier d'état civil.

Le pacs est doté d'un statut plus ténu que le mariage, les partenaires étant essentiellement soumis à des règles dordre patrimonial sans quexiste pour autant entre eux à l'instar du mariage un quelconque régime matrimonial. Par ailleurs, le pacs peut se rompre plus facilement que le mariage, les modes de dissolution du pacs étant plus nombreux et plus rapides que ceux du mariage et les conséquences d'une telle dissolution étant beaucoup moins protectrices qu'en matière de mariage (en particulier, absence de droit à prestation compensatoire ou de vocation successorale).

Enfin, le principe du pluralisme du couple conjugal se traduit par l'existence du concubinage, situation de fait non organisée par la loi et qui doit le rester pour ceux qui veulent s'aimer dans l'instant : c'est ce que l'on appelle aussi volontiers l'union libre! Bien que faisant l'objet d'une définition légale depuis la loi relative au PACS, la notion s'appliquant aussi bien aux couples d'homosexuels que d'hétérosexuels, il nentraîne l'application d’aucun statut général mais se voit appliquer de façon ponctuelle quelques effets juridiques : en matière de protection sociale par exemple ou encore par l'application de contrats de concubinage.

Il est ainsi impossible aujourd'hui de nier l'existence en droit français d'une trilogie des couples : mariage, pacs, concubinage, le couple étant une notion plurielle !

Si le droit français connaît ainsi plusieurs formes de conjugalité, il connaît aussi plusieurs formes de parenté.

\section{B. La parentalité}

La parentalité correspond à la fonction dêtre parent. La façon dont le droit français appréhende le couple parental a elle aussi beaucoup évolué au cours

16 J.-F SAGAUT, Notaires, à vos actes, prêts, pacsez !: Defrénois 2011, p. 1106.

17 V. AVENA-ROBARDET, Enregistrement du pacs notarié : AJ Famille 2012, p. 426.

(C) Palacký University Olomouc, Czech Republic, 2013. ISSN 1213-8770 (print), ISSN: 2464-6601 (online). 
de ces dernières années et fait l'objet d'une véritable révolution juridique avec les textes récents. La consécration d'une double parenté mono sexuée amène en effet à modifier totalement le droit de la filiation charnelle et de l'adoption.

Traditionnellement, la parenté est liée à la filiation par le sang, à la filiation biologique : elle assure le rattachement de l'enfant à un homme et une femme qui lont engendré. La présomption de paternité selon laquelle «lenfant conçu ou né pendant le mariage a pour père le mari de la mère » traduit d'ailleurs cette finalité procréative assignée initialement au mariage en facilitant de la sorte la preuve de la paternité du mari. Si la parenté a subi au départ l'attraction du mariage, force a été de constater le développement du nombre de naissances hors mariage pour modifier le droit de la filiation en prenant en compte ce phénomène : les enfants naturels se sont vus ainsi octroyés des droits équivalents à ceux des enfants légitimes et les couples de parents mariés et non mariés se sont vus attribués des droits équivalents dans leurs rapports avec leurs enfants.

Il est acquis toutefois que la parenté ne s'en tient pas à cette seule conception purement biologique. Elle peut aller au-delà et traduire des liens affectifs. Ainsi, depuis des temps déjà anciens, la filiation adoptive, créatrice d'une filiation purement élective répond à cet autre aspect de la notion de filiation. Elle permet de donner des parents à des enfants qui nen ont pas. Il est par ailleurs admis depuis longtemps que le lien sociologique créé par une filiation non immédiatement contestée pouvait prévaloir en particulier pour la paternité sur la vérité biologique, ceci par un jeu de présomptions et de brefs délais venant consolider juridiquement un lien biologiquement incertain (rôle fondamental à cet égard de la possession détat).

Parenté dans le mariage, parenté hors mariage, parenté liée à des relations charnelles ou même en dehors de toute relation charnelle, tels sont les différents visages des couples parentaux. Il est à remarquer à ce dernier titre que lévolution des techniques médicales ayant donné naissance aux lois bioéthique du 29 juillet 1994 modifiées en dernier lieu par la loi du 7 juillet 2011 a renforcé cette dissociation de la parenté et de toute relation charnelle en réglementant divers modes de procréation telle l'insémination artificielle ou la fécondation in vitro susceptibles de prévoir le recours à un ou plusieurs tiers donneurs.

Ces profondes mutations liées à la maitrise de la procréation ont certes ébranlé la vision traditionnelle de la parenté. Cependant, toutes les règles existant jusque là ont été élaborées sur la base d'une parenté hétérosexuelle, l'enfant devant être placé dans un système de parenté le rattachant à un père et à une mère, à un seul père et à une seule mère.

En consacrant une double parenté mono sexuée (l'homoparentalité), le droit contemporain fait vaciller les fondements du droit de la famille. La loi du 17 mai 2013 accorde en effet aux couples de même sexe l'accès à la parenté via le mécanisme de l'adoption. Le Conseil constitutionnel qui a eu à examiner le 
contenu de la loi au regard des principes fondamentaux de la république a à cet égard jugé que si le texte est conforme à la Constitution, il ne peut pas ouvrir un droit à l'adoption à tous, toute adoption reposant sur la recherche de l'intérêt de lenfant, principe ayant valeur constitutionnelle. La réforme n’a pas ainsi pour but de consacrer un droit à l'enfant en toutes circonstances ${ }^{18}$.

Pratiquement d'ailleurs, si l'adoption classique d'un enfant abandonné est visée, sa mise en œuvre ne sera pas évidente. Ladoption conjointe en matière d'adoption internationale apparait en effet délicate non seulement en raison du faible nombre d'enfants adoptables, mais aussi en raison du refus de nombreux pays de faire adopter leurs ressortissants par des couples homosexuels. Cest en fait l'adoption de l'enfant du conjoint qui est prioritairement revendiquée et qui sera vraisemblablement essentiellement pratiquée ${ }^{19}$. Or, ce type d'adoption obéit à une procédure simplifiée puisque la différence dâge entre adopté et adoptant est réduite, que l'enfant de moins de deux ans n’a pas à être remis préalablement au service d'aide sociale à l'enfance, et que le conjoint n’a pas besoin d'agrément. Dans les différents cas envisageables, une fois l'adoption prononcée, les deux parents détiendront l'autorité parentale. La réalité de l’adoption qui apparait ainsi en pratique assez limitée reste toutefois quant à son avenir étroitement liée au choix que le législateur fera demain en matière de PMA. A l'heure actuelle fermée aux couples de même sexe, elle n'empêche pas certaines femmes d'y recourir en marge de la loi. La question des PMA et de son corollaire, la gestation pour autrui a été renvoyée à un examen ultérieur ${ }^{20}$ !

C’est donc au nom d’une égalité très contestée que le législateur français multiplie les formes de couples parentaux, allant jusquà faire abstraction du sexe des êtres humains ${ }^{21}$. La parenté dans sa signification traditionnelle amenant à faire référence à un père et une mère apparaît aujourd'hui remise en cause.

Si l'on constate la reconnaissance progressive d'un droit pour les couples, le couple étant manifestement compris comme une notion plurielle susceptible de revêtir des formes diverses, il apparaît aussi paradoxalement quémerge au sein de cette diversité un droit des couples, une sorte de droit commun pour tous les couples.

18 C. NEIRINCK, Accorder le mariage aux personnes de même sexe, oui. Reconnaitre un droit à l'enfant, non ! Pourquoi ?: Dr. famille 2013, dossier 2; F. CHENEDE, La nouvelle leçon de démocratie du Conseil constitutionnel : AJ Famille 2013, p. 332.

19 F. BERDEAUX-GACOGNE, La discrète reconnaissance du parent social : AJ Famille 2013, p. 346.

20 I. CORPART, Le mariage pour tous et ses incidences sur le sort des enfants : AJ Famille 2013, p. 340.

21 H. FULCHIRON, Le mariage pour tous. Un enfant pour qui ? - Loi n²013-404 du 17 mai 2013: JCP G 2013, 658; C. NEIRINCK, Le mariage homosexuel ou l'arbre qui cache la forêt : Dr. famille 2012, repère 8 ; Faut-il tenir compte du sexe des êtres humains ? : Dr. famille 2012, repère 10. 


\section{L'EMERGENCE D'UN DROIT DES COUPLES}

Lémergence d'un socle commun de règles pour tous les couples a vocation à protéger l’ensemble des couples dans l'intérêt des familles.

Lavènement d'un droit commun du couple peut à vrai dire être constaté tant en ce qui concerne le couple dans ses rapports internes, c'est-à-dire le couple conjugal (A) qu'en ce qui concerne le couple dans ses rapports avec les enfants, c'est-à-dire le couple parental (B).

\section{A. Un droit pour le couple conjugal}

Bien que la conjugalité se décline aujourd'hui sous plusieurs formes, on peut déceler en droit français un droit de la formation du couple, certaines conditions de fond et de forme étant exigées à titre commun pour l'ensemble des couples.

L'interdit de l'inceste, le respect du principe monogamique et l'exigence d'un âge légal semblent ainsi constituer des conditions nécessaires à l'édification d'un couple. Par ailleurs, le mariage et le PACS supposent de façon commune au titre des conditions de forme la conclusion d'une convention et un passage devant l'autorité compétente (maire pour le mariage, greffier du tribunal d'instance ou notaire si le PACS revêt la forme d'un acte authentique depuis la loi du 28 mars 2011 de modernisation des professions judiciaires ou juridiques et certaines professions réglementées) qui doit enregistrer l'opération puis la transcrire sur le registre d'état civil. Et, si les candidats au mariage ou au PACS n'ont pas respecté les conditions de fond ou de forme requises par la loi pour la validité de l'acte, lopération pourra être déclarée nulle par le tribunal.

On peut constater par ailleurs un droit des effets du couple, la vie en couple supposant le respect d'un certain nombre d'obligations. Il s'agit non seulement de devoirs personnels comme l'obligation de vie commune ou d'assistance, mais aussi de devoirs matériels comme lobligation de contribuer aux charges de la vie commune (le JAF étant d'ailleurs compétent depuis la loi du 12 mai 2009 pour trancher les questions relatives à la contribution aux charges du mariage et $\mathrm{du}$ pacte). Pour le concubinage, la loi de 2009 permet aux concubins de saisir le juge aux fins de résoudre les difficultés liées au fonctionnement de l'indivision. D’ailleurs, rien n'interdit aux concubins de s'engager l'un envers l'autre à une telle contribution. En outre, face aux créanciers, les personnes qui vivent en couple doivent se montrer solidaires au moins pour les dettes de la vie courante (C. civ, art. 220 applicable traditionnellement aux époux et 515-4 pour les pacsés modifié par la loi du $1^{\mathrm{e}}$ juillet 2010 relative au crédit à la consommation).

La vie en couple suppose également le respect par chaque membre du couple de l'autre, ce qui exclut toute forme de violence. On peut signaler à cet égard l'existence de solutions communes à toutes les formes de conjugalité qui concernent aussi bien la matière pénale que civile. Les violences conjugales constituent sans

\footnotetext{
(C) Palacký University Olomouc, Czech Republic, 2013.

ISSN 1213-8770 (print), ISSN: 2464-6601 (online).
} 
doute en effet le fléau contemporain du droit commun de la famille. Une loi du 9 juillet 2010 relative aux violences faites spécifiquement aux femmes, aux violences au sein des couples et aux incidences de ces dernières sur les enfants octroie en ce sens au juge aux affaires familiales (JAF) des prérogatives civiles et pénales pour mettre à l'abri les victimes de violences au sein du couple qu'il soit marié ou non. Il est à remarquer que par ce texte, le JAF apparait une fois de plus comme le juge du couple sous toutes ses formes. Larticle 515-9 du Code civil permet notamment au juge aux affaires familiales de délivrer en urgence une ordonnance de protection qui peut porter notamment sur la résidence séparée des époux, pacsés ou concubins ou sur les modalités d'exercice de l'autorité parentale. Jusque là, le JAF nétait compétent que pour régler les crises du couple marié en application de l'article 220-1 du Code civil. Cela voulait dire que la concubine battue navait d'autre choix que de partir en ne pouvant en tout et pour tout quespérer une priorité pour l'attribution d'un logement social ; elle ne pouvait pas compter sur le juge civil. La loi nouvelle a mis fin à ces inégalités, le JAF pouvant désormais prendre, dans le cadre de lordonnance de protection toute une série d'interdictions pour mettre fin aux violences dans le couple et il peut statuer sur l'attribution du logement, la notion de logement familial concernant désormais tous les couples.

Il apparaît enfin que s'est mis en place progressivement un droit de la rupture du couple. On peut ainsi distinguer en la matière la rupture conventionnelle supposant un commun accord des membres du couple et des modes de rupture non conventionnelles incluant la dissolution par volonté unilatérale et la résiliation pour faute.

Lorsque la dissolution du couple résulte différemment du décès d'un des membres du couple, on trouve également dans la législation récente la volonté de protéger le survivant quelle que soit la forme de conjugalité. La loi du 3 décembre 2001 est venue à ce titre améliorer nettement la vocation successorale du conjoint survivant et consacrer l'existence d'un droit au logement ; par ailleurs, la loi TEPA du 21 août 2007 exonère le conjoint survivant de tout droit de succession $^{22}$. De façon comparable, le partenaire pacsé survivant se voit reconnaitre des droits sur le logement (droit temporaire au logement et attribution préférentielle possible du logement) et exonérer de droits de succession.

Comme il apparaît quémerge depuis quelques années un droit commun du couple conjugal, les mutations contemporaines du droit de la famille traduisent de façon comparable lémergence d'un droit commun du couple parental.

22 B. BEIGNIER, Libéralités et successions : éd. Monchrestien 2012, n 481 et s. p. 222 et s.; I. DAURIAC, Le conjoint survivant dans la loi du 23 juin 2006 : JCP N 2007, 1203. 


\section{B. Un droit pour le couple parental}

Lémergence d'un droit commun du couple parental amène à constater une homogénéité entre les différentes sortes de couples (mariés ou non mariés) dans les rapports avec leurs enfants.

La loi du 4 juin 1970 relative à l'autorité parentale est d'abord venue substituer à la notion de chef de famille celle d'autorité parentale qui induit une égalité entre les parents dans les rapports avec leurs enfants. Dans les conventions internationales, on parle plutôt de responsabilité parentale ${ }^{23}$. Or, cette responsabilité, c'est à travers la notion d'autorité parentale que le législateur l'a introduite en droit français en 1970 d'abord pour les couples mariés puis par la suite pour les couples non mariés.

Certaines lois postérieures à la loi de 1970 sont en effet venues ensuite consacrer le principe d'autorité parentale conjointe que les couples soient unis par les liens du mariage ou pas. Ce principe, contenue en dernier lieu dans la loi du 4 mars 2002 suppose l'exercice en commun de l'autorité parentale par les deux membres du couple. Le terme de coparentalité sert d'ailleurs souvent à désigner le couple parental et à exprimer ce principe d'exercice en commun de l'autorité parentale.

Appliqué tout naturellement aux couples qui vivent ensemble, la règle de coparentalité est également appliquée aux couples séparés. Toute une partie de la loi du 4 mars 2002 est en effet relative à «l'exercice de l'autorité parentale par les parents séparés ${ }^{24}$. La règle est que "la séparation des parents est sans incidence sur les règles de dévolution de l'autorité parentale ${ }^{25}$. Elle vaut tant pour les couples divorcés que pour les couples séparés qui nétaient pas mariés. Le législateur précise les deux obligations qui en découlent : d'une part l'obligation pour chaque parent de maintenir des relations avec l'enfant, d'autre part l'obligation pour chaque parent de respecter les liens de l'enfant avec l'autre parent. Et, pour assurer le maintien effectif du couple parental au-delà de la séparation, différentes mesures concrètes ont été imaginées par le législateur en sollicitant l'accord des parents pour favoriser un exercice consensuel de l'autorité parentale, en instaurant la possibilité d'une résidence alternée, en imposant aux parents une obligation d'information en cas de changement de résidence notamment.

\section{Les lois bioéthiques du 29 juillet 1994 modifiées en dernier lieu par la loi} du 7 juillet 2011 traduisent également l'émergence d'un droit commun pour le couple parental en admettant de façon semblable le recours aux méthodes de procréation médicalement assistée que le couple soit marié ou vive en concu-

23 Il résulte notamment de l'article 18-1 de la Convention relative aux droits de l'enfant dite Convention de New York que "Les Etats parties semploient de leur mieux à assurer la reconnaissance du principe selon lequel les deux parents ont une responsabilité commune pour ce qui est d'élever leur enfant et d'assurer son développement».

24 C.civ, art. 373-2 et s.

25 C.civ, art. 373-2 al. 1.

(c) Palacký University Olomouc, Czech Republic, 2013. ISSN 1213-8770 (print), ISSN: 2464-6601 (online). 
binage sans exiger désormais la condition antérieure d'une durée de vie commune de deux ans. A l'heure actuelle cependant, ces méthodes sont réservées aux couples composés d'un homme et d'une femme qui expriment une demande parentale. En revanche, même si certaines voix se font entendre en ce sens, la PMA nest pas ouverte aux couples homosexuels ${ }^{26}$.

Lévolution du droit français révèle en conséquence à quel point le couple ne lui est pas indifférent. Il l'intéresse en effet à un point tel qu'il tend à constituer un des piliers du droit de la famille contemporain lui-même en plein bouleversement. Il est devenu une unité à par entière car il remplit des fonctions utiles pour la société permettant notamment de lutter contre l'isolement, un des fléaux de notre époque !

Comme a pu lécrire Alphonse de Lamartine, poète et romancier français du XIXe siècle, "Toute saison, tout ciel sont bons quand on est deux ! ». Et Alain, Philosophe français bien connu du XXe siècle a rajouté «Aimer, ceest trouver sa richesse hors de soi ».

26 A. DIONISI-PEYRUSE, Brèves remarques sur la question de louverture de l'AMP aux couples de même sexe : AJ Famille 2013, p. 127. 